\title{
Regional anesthesia for pediatric knee surgery: a review of the indications, procedures, outcomes, safety, and challenges
}

This article was published in the following Dove Press journal:

Local and Regional Anesthesia

5 November 2015

Number of times this article has been viewed

\section{Wallis T Muhly \\ Harshad G Gurnaney \\ Arjunan Ganesh}

Department of Anesthesiology and Critical Care Medicine, The Children's Hospital of Philadelphia, Perelman School of Medicine at the University of Pennsylvania, Pennsylvania, PA, USA
Correspondence: Arjunan Ganesh Department of Anesthesiology and Critical Care Medicine,

The Children's Hospital of Philadelphia, 34th Street and Civic Center Boulevard, Philadelphia, PA 19104-4399, USA Email ganesha@email.chop.edu

\begin{abstract}
The indications for surgery on the knee in children and adolescents share some similarity to adult practice in that there are an increasing number of sports-related injuries requiring surgical repair. In addition, there are some unique age-related conditions or congenital abnormalities that may present as indications for orthopedic intervention at the level of the knee. The efficacy and safety of peripheral nerve blocks (PNBs) for postoperative analgesia following orthopedic surgery has been well established in adults. Recent studies have also demonstrated earlier functional recovery after surgery in patients who received PNBs. In children, PNB is gaining popularity, and increasing data are emerging to demonstrate the feasibility, efficacy, and safety in this population. In this paper, we will review some of the most common indications for surgery involving the knee in children and the anatomy of knee, associated dermatomal and osteotomal innervation, and the PNBs most commonly used to produce analgesia at the level of the knee. We will review the evidence in support of regional anesthesia in children in terms of both the quality conferred to the immediate postoperative care and the role of continuous PNBs in maintaining effective analgesia following discharge. Also we will discuss some of the subtle challenges in utilizing regional anesthesia in the pediatric patient including the use of general anesthesia when performing regional anesthesia and the issue of monitoring for compartment syndrome. Finally, we will offer some thoughts about areas of practice that are in need of further investigation.
\end{abstract}

Keywords: pediatric surgery, regional anesthesia, analgesia, knee surgery

\section{Surgical indications}

When managing the child with a knee injury or abnormality, it is critical that one considers the fact that, up until around ages of 12-14 years in girls and 14-16 years in boys, the growth plate remains open. Thus, any injury or surgical repair that impacts or causes early closure of the affected growth plate can lead to complications such as leg length discrepancy. With that in mind, one can begin to break down diseases of the knee into two general categories: congenitally or developmentally acquired diseases and traumatic injuries.

In the first category, a heterogeneous group of neuromuscular diseases, including cerebral palsy and arthrogryposis, can lead to limb abnormalities and distal femur angulation that can necessitate surgical correction. ${ }^{1-4}$ Surgery can include soft tissue tendon transfers, ligamentous lengthening or shortening, as well as more significant boney work that can include distal femoral osteotomy. This is often a medically fragile population with the potential for developmental delay and respiratory insufficiency. Use of regional anesthesia should be considered in this population either as a single 
injection for soft tissue work or continuous peripheral nerve block (CPNB) for significant osteotomies ${ }^{5}$ in an attempt to minimize opioid-related complications.

Abnormalities of the growth plate can present as either congenital or acquired valgus or varus deformities. Acquired varus deformities or Blount disease are commonly associated with obesity. ${ }^{6}$ The stress placed on medial compartment of the knee can lead to long-term mechanical damage and the potential for early onset joint dysfunction. Increasingly, these patients are initially managed with hemiepiphysiodesis techniques at the level of knee. ${ }^{7-9}$ Epiphysiodesis involves placement of implants including staples, transphyseal screws, or small plates across the faster growing physis to allow for temporary arrest of growth in attempt to restore normal joint angulation. These are typically same day surgeries and patients can benefit from single-injection femoral nerve blocks to limit opioid consumption and improve discharge readiness. Patients who fail to respond to conservative surgical interventions related to Blount disease may require tibial/fibular osteotomy for definitive correction. In these cases, a sciatic CPNB with or without a femoral CPNB may be of benefit. ${ }^{10}$

In the realm of sports medicine, a number of knee injuries and abnormalities in the chondral surface may require surgical intervention. ${ }^{11}$ Anterior cruciate ligament (ACL) injuries can present as primary ligamentous tears or less commonly avulsions secondary to tibial eminence fractures, and surgical repair is mandated to restore joint stability. Patellar dislocations, with associated medial patellofemoral ligament (MPFL) injury, that fail to heal with nonoperative treatment, may benefit from MPFL reconstruction. Finally, osteochondritis dissecans of the knee is an acquired, idiopathic, and potentially reversible disease of the subchondral bone that can lead to delamination and separation. ${ }^{11}$ Surgical treatment can range from simple arthroscopic drilling and removal of the lesion to open arthrotomy with chondral transplant for larger or unstable lesions. Depending on the magnitude of the surgery, single-injection femoral with or without sciatic block may be sufficient, while other larger surgeries including ACL and MPFL reconstruction or open arthrotomy for management of complex osteochondritis dissecans lesions may be better managed with femoral CPNB.

\section{Innervation of the knee and associated nerve blocks Anatomy and innervation}

A fundamental knowledge of the anatomical structure of the knee and associated neural innervation is critical to understand the utility and limitations of specific nerve blocks in managing postsurgical pain. Innervation of the knee comes from nerves arising from the lumbar plexus and the sciatic nerve.

The lumbar plexus lies within the substance of the psoas major muscle, anterior to the transverse processes of the lumbar vertebrae. It is formed by the first three, and a most of the fourth, lumbar ventral rami. The lumbar plexus lies between the anterior and the posterior masses of the psoas major in line with the intervertebral foramina. The branches of the plexus include the iliohypogastric, ilioinguinal, genitofemoral, lateral femoral cutaneous (LFC), femoral, and obturator nerves. $^{12}$

The femoral nerve descends through the psoas major and emerges at its lateral border. At the level of the inguinal ligament, it lies between the psoas major and iliacus muscle deep to the iliac fascia. Posterior to the inguinal ligament, the femoral nerve is lateral to the femoral artery and is separated from it by a part of the psoas major muscle. As the nerve passes into the thigh, it divides into an anterior and a posterior division and quickly arborizes. ${ }^{13}$

The obturator nerve forms within the substance of the psoas major from the anterior divisions of the second, third, and fourth lumbar nerves. It pierces the medial border of the psoas to reach the obturator foramen. ${ }^{14}$ The divergence of the femoral nerve from the obturator nerve begins as they emerge from the psoas major. At the level of the inguinal ligament, the obturator nerve is separated from the femoral nerve by several fascial compartments.

The LFC nerve is formed by the union of the posterior divisions of the anterior primary rami of L2 and L3. The LFC nerve perforates the inguinal ligament $\sim 1 \mathrm{~cm}$ from the anterior superior iliac spine and enters the thigh. ${ }^{15}$ This is a purely sensory nerve.

The sciatic nerve is formed of two components, tibial and common peroneal nerves. The tibial component is formed by the union of L4-L5 and the anterior divisions of the sacral plexus (S1-S3), whereas the common peroneal component is formed by the union of the posterior divisions of the same nerve roots (L4-S3). The sciatic nerve exits the pelvis via the greater sciatic notch. At this level, the sciatic nerve lies lateral and posterior to the ischial spine. In the thigh, the nerve is posterior to the lesser trochanter of the femur on the posterior surface of the adductor magnus and deep to the biceps femoris. ${ }^{16}$ In the upper part of the popliteal fossa, the sciatic nerve lies posterolateral to the popliteal vessels. The sciatic nerve divides into the two components in the popliteal fossa. ${ }^{17}$ The tibial branch continues along the tibialis posterior muscle with the posterior tibial vessels. 
The common peroneal nerve diverges laterally in the popliteal fossa and lies subcutaneously just behind the fibular head where it is prone to injury. The common peroneal then divides into superficial and deep peroneal nerves.

The femoral nerve innervates the dermatomes over the anterior thigh and knee, while the LFC nerve innervates the skin over the lateral aspects of the thigh and the knee. The posterior cutaneous nerve of the thigh, which is a branch of the sciatic nerve, innervates the skin over the posterior aspect of the knee. The femoral nerve innervates the majority of the femur bone. In a small percentage of individuals, the obturator nerve innervates the medial portion of the femur close to the knee joint. The knee joint is innervated by two distinct groups of afferent nerves. The anterior group of afferent nerves includes the articular branches of the femoral, common peroneal, and saphenous nerves. The posterior group includes the posterior articular branch of the tibial nerve and sometimes a branch from the obturator nerves. ${ }^{18}$

\section{Block techniques Femoral nerve block}

Indications: This block is used for perioperative analgesia for femur fractures, surgery on the anterior thigh, arthroscopy of the knee, ACL reconstruction, and total knee arthroplasty (TKA) as part of a multimodal regimen.

Contraindications: Patient refusal is an absolute contraindication to the placement of PNB. Other contraindications are infection at the site of needle placement, allergy to local anesthetic, preexisting peripheral neuropathy, and severe neurologic injury, which precludes postoperative assessment for complications.

Landmarks: The femoral nerve block is performed $\sim 1 \mathrm{~cm}$ below the inguinal ligament in the thigh. The nerve is lateral and posterior to the femoral artery. ${ }^{19}$

Ultrasound technique: The femoral nerve divides into an anterior and a posterior division in the groin region. For knee procedures, the posterior division is important to block. This is identified by stimulation of the quadriceps muscle using the nerve stimulator. With the patient in the supine position, the needle entry is at a point $1 \mathrm{~cm}$ below the inguinal ligament and $1 \mathrm{~cm}$ lateral to the femoral artery. The needle is placed with the nerve stimulator set at $1 \mathrm{~mA}$ to identify the femoral nerve. The needle position is adjusted to achieve stimulation around $0.5 \mathrm{~mA}$ prior to injection of the local anesthetic.

Dose of local anesthetic: $1 \mathrm{~mL} / \mathrm{kg}$ of $0.125 \%$ bupivacaine or $0.2 \%$ ropivacaine up to a maximum of $30 \mathrm{~mL}$.

Complications: Injury to the adjoining structures, notably the femoral vessels. Infection of the injection site and peripheral neuropathy are also complications of the femoral nerve block.

\section{Adductor canal block}

Indications: This block is used for perioperative analgesia for surgery on the distal anterior thigh, arthroscopy of the knee, and TKA as part of a multimodal regimen. ${ }^{20}$

Contraindications: Contraindications are similar to that for a femoral nerve block.

Landmarks: The saphenous branch of the femoral nerve is identified using ultrasound guidance on the medial part of the mid-thigh at the midpoint between the inguinal crease and the patella.

Ultrasound technique: The saphenous branch of the femoral nerve is identified on the medical aspect of the mid-thigh. Using a short axis (transverse view), the femoral artery is identified under the sartorius muscle. The saphenous vein lies inferior to the artery in the adductor canal and the nerve is visualized as a hyperechoic structure anterior or lateral to the femoral artery.

Dose of local anesthetic: $1 \mathrm{~mL} / \mathrm{kg}$ of $0.125 \%$ bupivacaine or $0.2 \%$ ropivacaine up to a maximum of $30 \mathrm{~mL}$ can be used for this block. Larger volumes may be associated with an increased risk for a motor block in the distribution of the femoral nerve.

Complications: Injury to the adjoining structures, notably the femoral vessels. The risk of a motor block of the adductor and quadriceps muscle is lower than that of a femoral nerve block but still exists.

\section{Sciatic nerve block}

The sciatic nerve block can be placed at either the subgluteal or a mid-thigh location for knee procedures depending on the type of procedure.

\section{Sciatic nerve block (subgluteal approach)}

Indications: Subgluteal sciatic nerve block is indicated for knee surgery, which also involves the posterior thigh (eg, hamstring autograft for ACL repair).

Contraindications: Contraindications are similar to that for a femoral nerve block.

Landmarks: Anatomical landmarks include the greater trochanter, posterior superior iliac spine, ischial tuberosity, and the sacral hiatus.

Technique: For this approach (subgluteal), the patient is positioned in the lateral decubitus position with the nonoperative side down. The greater trochanter and the ischial tuberosity are identified. At the midpoint of this line, a perpendicular line is extended caudally for $4 \mathrm{~cm}$ where a groove 
between the biceps femoris and the semitendinosus muscles can be palpated. The stimulating needle is introduced at an angle of $80^{\circ}$ to the skin with stimulation at $1.5 \mathrm{~mA}, 2 \mathrm{~Hz}$, and $0.1 \mathrm{~ms}$. Once the sciatic nerve is located, the needle position is adjusted to achieve stimulation around $0.5 \mathrm{~mA}$ prior to injection of the local anesthetic.

Ultrasound guided technique: The sciatic nerve can be clearly identified in the above-mentioned approaches using a high-resolution ultrasound probe, and the anesthetic can be injected under direct ultrasound guidance. ${ }^{21}$

Dose of local anesthetic: $0.5 \mathrm{~mL} / \mathrm{kg}$ of $0.125 \%$ bupivacaine or $0.15 \%$ ropivacaine up to a maximum of $20 \mathrm{~mL}$ can be used.

\section{Sciatic nerve block (mid-thigh approach)}

Indications: This sciatic nerve block is indicated for knee procedures not involving a tendon harvest from the hamstrings.

Landmarks:

Posterior approach: Lateral border of the biceps femoris and medial border of the semi-membranous and semitendinosus tendons.

Lateral approach: The groove between the biceps femoris and the vastus lateralis on the lateral aspect of the thigh.

Technique: For the posterior approach, the patient is positioned prone. The entry point for the needle is high in the popliteal fossa between the semi-membranous and biceps femoris tendons and medial to the biceps femoris tendon. The needle is introduced perpendicular to the skin and gradually advanced until the sciatic nerve is stimulated. This is identified by a motor response in either branches of the sciatic nerve, that is, common peroneal nerve (identified by dorsiflexion or eversion of the foot) or posterior tibial nerve (identified by plantar flexion and inversion); the needle is positioned to reduce the stimulating current to $\sim 0.5 \mathrm{~mA}$ prior to the local anesthetic administration.

For the lateral approach, the patient is placed in the supine position and the leg elevated so the foot can move freely. The entry point for the needle is in the groove between the vastus lateralis and the biceps femoris muscles $\sim 10 \mathrm{~cm}$ cephalad to the lateral femoral condyle. The needle is directed at a $30^{\circ}$ angle posterior to the horizontal plane until motor response seen in the distribution of the sciatic nerve is stimulated. The needle is positioned to reduce the stimulating current to $\sim 0.5 \mathrm{~mA}$ prior to injection of the local anesthetic.

Dose of local anesthetic: $0.5 \mathrm{~mL} / \mathrm{kg}$ of $0.125 \%$ bupivacaine or $0.15 \%$ ropivacaine up to a maximum of $20 \mathrm{~mL}$ can be used.

\section{Continuous peripheral nerve infusion Local anesthetics and additives}

Ropivacaine and bupivacaine are the most commonly used local anesthetics for PNB in children. Ropivacaine by virtue of its slightly better safety profile is increasingly replacing bupivacaine as the local anesthetic of choice for PNB. ${ }^{22,23} \mathrm{We}$ now exclusively use ropivacaine for PNB in our practice. As opposed to practice in adult patients, lower concentrations of local anesthetics suffice in children $(0.1 \%-0.2 \%)$, as almost all procedures are done under general anesthesia. Use of lower concentrations of local anesthetics provides adequate sensory block for postoperative analgesia and lowers the incidence of motor blockade. In addition, it also decreases the total dose administered and consequently the potential for local anesthetic toxicity.

Several different additives to local anesthetics have been tried to increase the duration and the quality of analgesia. Addition of clonidine has been shown to increase the duration of PNB in children. ${ }^{24}$

\section{Postoperative monitoring}

Detailed instructions should be provided to all patients who are discharged home after a PNB that has not completely resolved. Instructions should include protection of the insensate extremity and avoidance of independent ambulation with or without the use of support (crutches, walker) until complete return of motor function. All patients should be provided with an expected duration for which their PNB will last, along with instructions for management of pain when the nerve block wears off. Instructions should also be provided for potential side effects (neurological signs or discharge/ redness at the site of the nerve block/catheter). Parents should be instructed to clamp the continuous infusion catheter for any change in neurologic status and that they should call a clinician from the anesthesia department/pain management service to discuss their child's symptoms. ${ }^{25}$

\section{Evidence and outcomes}

PNBs for pediatric knee surgery are emerging as a safe and effective technique for postoperative pain management. Several studies in children have demonstrated the safety, feasibility, and efficacy of PNB. ${ }^{26-34}$ The advantages of PNBs include efficient, site-specific analgesia, a decrease in the need for opioids, and consequently a decrease in opioidrelated side effects. The most common PNB performed in children are single-injection blocks. ${ }^{34,35} \mathrm{CPNBs}$ are performed in cases where significant analgesia is required for periods 
exceeding 12-24 hours. However, additional expertise is required to perform PNB and CPNB. In addition, if one is contemplating an ambulatory CPNB program, good patient and family education along with close follow-up are vital to ensure success and safety. ${ }^{29}$

Despite the growing evidence in support of regional anesthesia in children, there is a paucity of conclusive evidence demonstrating improved outcome compared to standard therapy. ${ }^{36}$ Several series have been published in pediatric populations that demonstrate clinical benefit with the use of CPNB in patients undergoing surgery on the knee. .,27,37,38 $^{5}$ However, level-one evidence, in the form of randomized control trials, is noticeably absent in the pediatric literature. Looking at the adult literature, we find a number of studies that can inform our understanding of pain management following knee surgery, but much of the adult literature has focused on TKA. ${ }^{39}$ Because TKA is relatively uncommon in pediatric populations, we will highlight data that support the use of regional anesthesia for ACL reconstruction, as this is a growing orthopedic issue in pediatric patients. ${ }^{11}$ Single-injection femoral nerve blocks have been shown to be effective compared to placebo in adult patients undergoing ACL reconstruction. ${ }^{40-42}$ Williams et al randomized patients undergoing ACL reconstruction to placebo bolus with sham catheter, levobupivacaine bolus with sham catheter, and levobupivacaine bolus with levobupivacaine infusion. The authors found that the femoral CPNB reliably kept pain scores lower than the moderate-tosevere pain threshold for the first 4 days after surgery. ${ }^{43}$

Increasingly, there have been concerns about the motor blockade associated with femoral nerve blockade in the TKA ${ }^{44}$ population, and, as a result, the ACL population gave the increased fall risk in this primarily ambulatory population. Some authors have found good pain control with infrapatellar nerve blockade as part of a multimodal perioperative analgesia approach, ${ }^{45}$ while other evidence suggests that saphenous block may be sufficient in ACL reconstruction with patellar graft. ${ }^{46}$ However, the causality between falls and muscle weakness during or following a nerve block in the setting of major knee surgery remains an area of debate. There is little evidence to suggest that regional anesthesia has an impact on functionality following ACL beyond the early postoperative period. A recent study of patients managed with femoral CPNB for 24 hours following TKA found that patients with postoperative femoral $\mathrm{CPNB}$ had improved range of motion at 1 month compared to patients managed with oral analgesics. ${ }^{47}$ Conversely, a recent retrospective study found that children and adolescents undergoing ACL reconstruction had persistent strength deficits and a delay in return to sports at 6 months in patients who were managed with a femoral nerve block compared to those who did not. ${ }^{48}$

\section{Controversies and future directions Regional under general anesthesia}

With the publication of a case report describing permanent neurological injury following epidural catheter placement ${ }^{49}$ in the anesthetized patient, there were general advisories made against performing regional anesthesia in the setting of a general anesthetic despite the protests of pediatric anesthesiologists. ${ }^{50}$ Similar controversy developed in a case of permanent loss of cervical spinal cord function following performance of an interscalene block $^{51}$ under general anesthesia. In the intervening years, more data have been presented demonstrating the safety of both neuraxial anesthesia and $\mathrm{PNBs}^{30,34,52,53}$ in children. The Pediatric Regional Anesthesia Network looked specifically at the question of asleep versus awake or sedated patients and regional anesthesia in $>50,000$ cases and found no difference in block-related neurological complications. ${ }^{54}$ Additionally, several reports have been published on the safety of interscalene blocks in children under general anesthesia ${ }^{55,56}$ without any major neurological complications or severe adverse events. Ultimately, safety is challenging to prove, but it may be that, with advent of better regional anesthesia training and the use of ultrasound for continuous visualization of the needle, regional anesthesia in the asleep patient should be considered an acceptable practice.

\section{PNBs and compartment syndrome}

Compartment syndrome is a rare and potentially devastating complication. Historically, there has been debate about using regional anesthesia in situations where compartment syndrome is a concern. Despite these concerns, there have been several compelling case reports of continuous lower extremity PNBs that present as increasing pain despite the presence of functioning nerve block. ${ }^{57-59}$ Although this evidence is not definitive, it seems that the ischemic pain, which is characteristic of acute compartment syndrome, is not completely ablated in the setting of peripheral nerve blockade. The ischemic pain signal may be transmitted via sympathetic pathways associated with the vasculature. ${ }^{57,60}$ Despite this theoretical evidence that regional anesthesia may not actually mask or delay the diagnosis of an evolving compartment syndrome, a high level of vigilance on the part of the surgical and anesthesia team is required to ensure that patients with PNBs have an appropriate level of postoperative monitoring to allow for early detection of this rare complication. 


\section{Future directions}

Much progress has been made in the expansion of regional anesthesia in the care of children and adolescents. When considering regional anesthesia for knee surgery, we have evidenced that it can be done safely and effectively but the evidence demonstrating improved overall outcomes is limited. We may be beyond the point of comparing regional anesthesia to opioid-based techniques, but important questions about the value of catheters compared to singleinjection blocks or the role of adjunctive agents in pediatric regional anesthesia are areas of potential investigation. Finally, it seems that investigations that extend beyond the perioperative period and 1st week following surgery are needed as we attempt to generate evidence supporting the use of regional anesthesia as an anesthetic adjunct in the management of pediatric knee surgery. While better early pain control is a very important goal, we must be sure that early or potentially late effects of regional anesthesia on muscle strength do not adversely impact patient outcomes.

\section{Disclosure}

The authors report no conflicts of interest in this work.

\section{References}

1. Novacheck TF, Stout JL, Gage JR, Schwartz MH. Distal femoral extension osteotomy and patellar tendon advancement to treat persistent crouch gait in cerebral palsy. Surgical technique. J Bone Joint Surg Am. 2009;91(Supp1 2):271-286.

2. Karol LA. Surgical management of the lower extremity in ambulatory children with cerebral palsy. J Am Acad Orthop Surg. 2004;12(3): 196-203.

3. Young JL, Rodda J, Selber P, Rutz E, Graham HK. Management of the knee in spastic diplegia: what is the dose? Orthop Clin North Am. 2010;41(4):561-577.

4. Lampasi M, Antonioli D, Donzelli O. Management of knee deformities in children with arthrogryposis. Musculoskelet Surg. 2012;96(3):161-169.

5. Dadure C, Bringuier S, Raux O, et al. Continuous peripheral nerve blocks for postoperative analgesia in children: feasibility and side effects in a cohort study of 339 catheters. Can J Anaesth. 2009;56(11): 843-850.

6. Sabharwal S. Blount disease: an update. Orthop Clin North Am. 2015; 46(1):37-47.

7. McIntosh AL, Hanson CM, Rathjen KE. Treatment of adolescent tibia vara with hemiepiphysiodesis: risk factors for failure. J Bone Joint Surg Am. 2009;91(12):2873-2879.

8. Westberry DE, Davids JR, Pugh LI, Blackhurst D. Tibia vara: results of hemiepiphyseodesis. J Pediatr Orthop B. 2004;13(6):374-378.

9. Wiemann JM, Tryon C, Szalay EA. Physeal stapling versus 8-plate hemiepiphysiodesis for guided correction of angular deformity about the knee. J Pediatr Orthop. 2009;29(5):481-485.

10. Muhly W, Gurnaney H, Hosalkar H, Kraemer F, Davidson R, Ganesh A. Continuous perineural infusion after lower extremity osteotomies in children: a feasibility and safety analysis. Br J Anaesth. 2013;110(5): 851-852.

11. Beck NA, Patel NM, Ganley TJ. The pediatric knee: current concepts in sports medicine. J Pediatr Orthop B. 2014;23(1):59-66.
12. Reinpold W, Schroeder AD, Schroeder M, Berger C, Rohr M, Wehrenberg U. Retroperitoneal anatomy of the iliohypogastric, ilioinguinal, genitofemoral, and lateral femoral cutaneous nerve: consequences for prevention and treatment of chronic inguinodynia. Hernia. 2015;19(4):539-548.

13. Lonchena TK, McFadden K, Orebaugh SL. Correlation of ultrasound appearance, gross anatomy, and histology of the femoral nerve at the femoral triangle. Surg Radiol Anat. 2015.

14. Anagnostopoulou S, Kostopanagiotou G, Paraskeuopoulos T, Alevizou A, Saranteas T. Obturator nerve block: from anatomy to ultrasound guidance. Anesth Analg. 2008;106(1):350. [author reply 350-351].

15. Murata Y, Takahashi K, Yamagata M, Shimada Y, Moriya H. The anatomy of the lateral femoral cutaneous nerve, with special reference to the harvesting of iliac bone graft. J Bone Joint Surg Am. 2000;82(5):746-747.

16. Moayeri N, van Geffen GJ, Bruhn J, Chan VW, Groen GJ. Correlation among ultrasound, cross-sectional anatomy, and histology of the sciatic nerve: a review. Reg Anesth Pain Med. 2010;35(5):442-449.

17. Vloka JD, Hadzic A, April E, Thys DM. The division of the sciatic nerve in the popliteal fossa: anatomical implications for popliteal nerve blockade. Anesth Analg. 2001;92(1):215-217.

18. Horner G, Dellon AL. Innervation of the human knee joint and implications for surgery. Clin Orthop Relat Res. 1994;301:221-226.

19. Orebaugh SL. The femoral nerve and its relationship to the lateral circumflex femoral artery. Anesth Analg. 2006;102(6):1859-1862.

20. Jæger P, Koscielniak-Nielsen ZJ, Schrøder HM, et al. Adductor canal block for postoperative pain treatment after revision knee arthroplasty: a blinded, randomized, placebo-controlled study. PLoS One. 2014; 9(11): e111951.

21. Ponde VC, Desai AP, Shah DM, Johari AN. Feasibility and efficacy of placement of continuous sciatic perineural catheters solely under ultrasound guidance in children: a descriptive study. Paediatr Anaesth. 2011;21(4):406-410.

22. Buckenmaier CC 3rd, Bleckner LL. Anaesthetic agents for advanced regional anaesthesia: a North American perspective. Drugs. 2005;65(6): 745-759.

23. Graf BM. The cardiotoxicity of local anesthetics: the place of ropivacaine. Curr Top Med Chem. 2001;1(3):207-214.

24. Cucchiaro G, Ganesh A. The effects of clonidine on postoperative analgesia after peripheral nerve blockade in children. Anesth Analg. 2007;104(3):532-537.

25. Gurnaney HG, Maxwell LG, Kraemer FW, Goebel T, Nance ML, Ganesh A. Prospective randomized observer-blinded study comparing the analgesic efficacy of ultrasound-guided rectus sheath block and local anaesthetic infiltration for umbilical hernia repair. Br J Anaesth. 2011;107(5):790-795.

26. Dadure C, Capdevila X. Continuous peripheral nerve blocks in children. Best Pract Res Clin Anaesthesiol. 2005;19(2):309-321.

27. Dadure C, Pirat P, Raux O, et al. Perioperative continuous peripheral nerve blocks with disposable infusion pumps in children: a prospective descriptive study. Anesth Analg. 2003;97(3):687-690.

28. Ganesh A, Cucchiaro G. Multiple simultaneous perineural infusions for postoperative analgesia in adolescents in an outpatient setting. $\mathrm{Br} J$ Anaesth. 2007;98(5):687-689.

29. Ganesh A, Rose JB, Wells L, et al. Continuous peripheral nerve blockade for inpatient and outpatient postoperative analgesia in children. Anesth Analg. 2007;105(5):1234-1242. [table of contents]

30. Giaufre E, Dalens B, Gombert A. Epidemiology and morbidity of regional anesthesia in children: a one-year prospective survey of the French-Language Society of Pediatric Anesthesiologists. Anesth Analg. 1996;83(5):904-912.

31. Ludot H, Berger J, Pichenot V, Belouadah M, Madi K, Malinovsky JM. Continuous peripheral nerve block for postoperative pain control at home: a prospective feasibility study in children. Reg Anesth Pain Med. 2008;33(1):52-56.

32. Rochette A, Dadure C, Raux O, Troncin R, Mailhee P, Capdevila X. A review of pediatric regional anesthesia practice during a 17-year period in a single institution. Paediatr Anaesth. 2007;17(9):874-880. 
33. van Geffen GJ, Gielen M. Ultrasound-guided subgluteal sciatic nerve blocks with stimulating catheters in children: a descriptive study. Anesth Analg. 2006;103(2):328-333. [table of contents].

34. Polaner DM, Taenzer AH, Walker BJ, et al. Pediatric Regional Anesthesia Network (PRAN): a multi-institutional study of the use and incidence of complications of pediatric regional anesthesia. Anesth Analg. 2012; 115(6):1353-1364

35. Ecoffey C. Pediatric regional anesthesia-update. Curr Opin Anaesthesiol. 2007;20(3):232-235.

36. Suresh S, Schaldenbrand K, Wallis B, De Oliveira GS Jr. Regional anaesthesia to improve pain outcomes in paediatric surgical patients: a qualitative systematic review of randomized controlled trials. $\mathrm{Br} J$ Anaesth. 2014;113(3):375-390.

37. Gurnaney H, Kraemer FW, Maxwell L, Muhly WT, Schleelein L, Ganesh A. Ambulatory continuous peripheral nerve blocks in children and adolescents: a longitudinal 8-year single center study. Anesth Analg. 2014;118(3):621-627.

38. Schloss B, Bhalla T, Klingele K, Phillips D, Prestwich B, Tobias JD. A retrospective review of femoral nerve block for postoperative analgesia after knee surgery in the pediatric population. $J$ Pediatr Orthop. 2014;34(4):459-461.

39. Xu J, Chen XM, Ma CK, Wang XR. Peripheral nerve blocks for postoperative pain after major knee surgery. Cochrane Database Syst Rev. 2014;12:CD010937.

40. Mulroy MF, Larkin KL, Batra MS, Hodgson PS, Owens BD. Femoral nerve block with $0.25 \%$ or $0.5 \%$ bupivacaine improves postoperative analgesia following outpatient arthroscopic anterior cruciate ligament repair. Reg Anesth Pain Med. 2001;26(1):24-29.

41. Wulf H, Lowe J, Gnutzmann KH, Steinfeldt T. Femoral nerve block with ropivacaine or bupivacaine in day case anterior crucial ligament reconstruction. Acta Anaesthesiol Scand. 2010;54(4):414-420.

42. Matava MJ, Prickett WD, Khodamoradi S, Abe S, Garbutt J. Femoral nerve blockade as a preemptive anesthetic in patients undergoing anterior cruciate ligament reconstruction: a prospective, randomized, double-blinded, placebo-controlled study. Am J Sports Med. 2009;37(1): 78-86.

43. Williams BA, Kentor ML, Vogt MT, et al. Reduction of verbal pain scores after anterior cruciate ligament reconstruction with 2-day continuous femoral nerve block: a randomized clinical trial. Anesthesiology. 2006;104(2):315-327.

44. Kandasami M, Kinninmonth AW, Sarungi M, Baines J, Scott NB. Femoral nerve block for total knee replacement - a word of caution. Knee. 2009;16(2):98-100.

45. Lundblad M, Forssblad M, Eksborg S, Lonnqvist PA. Ultrasound-guided infrapatellar nerve block for anterior cruciate ligament repair: a prospective, randomised, double-blind, placebo-controlled clinical trial. Eur J Anaesthesiol. 2011;28(7):511-518.

46. Chisholm MF, Bang H, Maalouf DB, et al. Postoperative analgesia with saphenous block appears equivalent to femoral nerve block in ACL reconstruction. HSS J. 2014;10(3):245-251.
47. Nader A, Kendall MC, Wixson RL, Chung B, Polakow LM, McCarthy RJ. A randomized trial of epidural analgesia followed by continuous femoral analgesia compared with oral opioid analgesia on short- and long-term functional recovery after total knee replacement. Pain Med. 2012;13(7):937-947.

48. Luo TD, Ashraf A, Dahm DL, Stuart MJ, McIntosh AL. Femoral nerve block is associated with persistent strength deficits at 6 months after anterior cruciate ligament reconstruction in pediatric and adolescent patients. Am J Sports Med. 2015;43(2):331-336.

49. Bromage PR, Benumof JL. Paraplegia following intracord injection during attempted epidural anesthesia under general anesthesia. Reg Anesth Pain Med. 1998;23(1):104-107.

50. Krane EJ, Dalens BJ, Murat I, Murrell D. The safety of epidurals placed during general anesthesia. Reg Anesth Pain Med. 1998;23(5):433-438.

51. Benumof JL. Permanent loss of cervical spinal cord function associated with interscalene block performed under general anesthesia. Anesthesiology. 2000;93(6):1541-1544.

52. Ecoffey C, Lacroix F, Giaufre E, Orliaguet G, Courreges P. Epidemiology and morbidity of regional anesthesia in children: a follow-up one-year prospective survey of the French-Language Society of Paediatric Anaesthesiologists (ADARPEF). Paediatr Anaesth. 2010;20(12):1061-1069.

53. Llewellyn N, Moriarty A. The national pediatric epidural audit. Paediatr Anaesth. 2007;17(6):520-533.

54. Taenzer AH, Walker BJ, Bosenberg AT, et al. Asleep versus awake: does it matter?: pediatric regional block complications by patient state: a report from the Pediatric Regional Anesthesia Network. Reg Anesth Pain Med. 2014;39(4):279-283.

55. Gurnaney H, Muhly WT, Kraemer FW, Cucchiaro G, Ganesh A. Safety of pediatric continuous interscalene block catheters placed under general anesthesia: a single center's experience. Acta Anaesthesiol Scand. 2015;59(3):377-383.

56. Taenzer A, Walker BJ, Bosenberg AT, et al. Interscalene brachial plexus blocks under general anesthesia in children: is this safe practice?: a report from the pediatric regional anesthesia network (PRAN). Reg Anesth Pain Med. 2014;39(6):502-505.

57. Kucera TJ, Boezaart AP. Regional anesthesia does not consistently block ischemic pain: two further cases and a review of the literature. Pain Med. 2014;15(2):316-319.

58. Walker BJ, Noonan KJ, Bosenberg AT. Evolving compartment syndrome not masked by a continuous peripheral nerve block: evidence-based case management. Reg Anesth Pain Med. 2012;37(4):393-397.

59. Cometa MA, Esch AT, Boezaart AP. Did continuous femoral and sciatic nerve block obscure the diagnosis or delay the treatment of acute lower leg compartment syndrome? A case report. Pain Med. 2011;12(5):823-828.

60. Frolich MA, Deshpande H, Ness T, Deutsch G. Quantitative changes in regional cerebral blood flow induced by cold, heat and ischemic pain: a continuous arterial spin labeling study. Anesthesiology. 2012. 117(4):857-867.
Local and Regional Anesthesia

\section{Publish your work in this journal}

Local and Regional Anesthesia is an international, peer-reviewed, open access journal publishing on the development, pharmacology, delivery and targeting and clinical use of local and regional anesthetics and analgesics. The journal welcomes submitted papers covering original research, basic science, clinical studies, reviews \& evaluations,

\section{Dovepress}

guidelines, expert opinion and commentary, case reports and extended reports. The manuscript management system is completely online and includes a very quick and fair peer-review system, which is all easy to use. Visit http://www.dovepress.com/testimonials.php to read real quotes from published authors. 